\title{
Radionuclide bone scanning in females with chronic low back pain
}

\author{
R. S. ROTHWELL, P. DAVIS, AND B. C. LENTLE
}

From the Rheumatic Disease Unit, Department of Medicine, University of Alberta, and the W.W. Cross Cancer Institute, Edmonton, Alberta, Canada

SUMMARY Sixty female patients with chronic low back pain have been studied clinically, radiographically, and by radionuclide bone scanning for evidence of sacroiliac disease. Twenty-four patients had quantitative sacroiliac scintigraphy (QSS) results suggesting sacroiliitis. In only one of these patients was the radiograph abnormal. Clinical and laboratory examinations failed to reveal any possible associated aetiological factors. Six-month follow-up of 18 patients showed that subjective improvement of pain is associated with a return to normal of QSS results, often secondary to anti-inflammatory medication. It is concluded that sacroiliac disease may be a common cause for chronic low back pain in women and that its presence may be missed if radiographs are relied upon to confirm the diagnosis. Its aetiology remains obscure.

Low backache is a common complaint in women presenting not only to family practitioners but also to gynecologists, orthopedic surgeons, and rheumatologists. Although many discrete entities, including sacroiliac disease, can be identified as the source of low back pain, a large group of patients exist whose vague symptoms, minimal signs, and normal laboratory studies generate such diagnoses as mechanical backache, lumbar strain, or psychogenic rheumatism.

Radiographic change can confirm a diagnosis of sacroiliac disease, but the absence of change does not rule out disease activity in these joints. Recently, the use of $99 \mathrm{~m}$ Tc stannous pyrophosphate bone scanning and quantitative sacroiliac scintigraphy (QSS) has been advocated as a useful adjunct to radiography in the assessment of sacroiliac disease. ${ }^{1-3}$

Consequently, the problem of chronic low backache in women was studied with scanning techniques in this rheumatic disease unit. ${ }^{4}$ This study showed that QSS is a useful means for detecting sacroiliac disease when radiographs are normal and that disorders of these joints are a common cause of low backache in women.

In this paper we have undertaken to assess possible clinical, laboratory, and radiographic correlates of sacroiliac disease as measured by quantitative sacro-

Accepted for publication 8 January 1980

Correspondence to Dr P. Davis, 9-112 Clinical Sciences Building, University of Alberta, Edmonton, Alberta T6G 2G3, Canada. iliac scintigraphy. In addition we have followed the evolution of the bone scan in several patients, including those who remain symptomatic and those who become pain-free.

\section{Patients and methods}

Sixty women, who were seen consecutively, were enrolled in this prospective study. The only criterion for inclusion was a presenting symptom of chronic low back pain of greater than 3 months' duration. A clinical history was obtained, including a review of the musculoskeletal system, obstetrical events, and significant gastrointestinal and genitourinary symptoms. A complete clinical examination was performed and focused on the lumbosacral spine. It involved assessment of configuration, tenderness, and range of motion (including a Schober test). In addition the clinical assessment included a search for sacroiliac inflammation by joint-stressing techniques, such as significant pressure applied in the lateral decubitus position. The hips were examined clinically for evidence of painful or restricted movement, and leg lengths were measured. Radiographs were obtained on all patients and included views of the pelvis, sacroiliac joints, and lumbosacral spine. The radiographs of the sacroiliac joints were read blind and were graded in accordance with the New York criteria. ${ }^{5}$ Laboratory studies performed included a complete blood count, erythrocyte sedimentation rate, biochemical profile, rheumatoid factor, and a 
urine analysis. All patients were HLA-typed. Radionuclide scintigraphy was performed by the following technique. ${ }^{99 \mathrm{~m}}$ Tc-labelled stannous pyrophosphate in a dosage of $175 \mu \mathrm{Ci}$ per kilogram was administered to obtain a 3-hour delayed bone scan. Quantification of the scan results was obtained as previously reported ${ }^{1}$ to reflect the ratio of radioisotope uptake in the sacroiliac joint to the uptake in the adjacent sacrum.

\section{Results}

In this study scintiscan results obtained in the 60 symptomatic patients were compared with those from 66 female controls who had had bone scans for reasons other than backache. The control group had a mean age of 31 (range 21-60) years and their scintiscan results, sacroliac: sacral ratios, (SI:S) showed a mean of $1 \cdot 18: 1$ ( \pm 2 SD being $0 \cdot 96: 1$ to $1 \cdot 40: 1)$. Consequently, a ratio of greater than 1.40:1 was taken as abnormal.

The 60 symptomatic patients had a mean age of 38 (range 18-69) years, and mean uptake ratios of 1.30:1 ( \pm 2 SD being $0 \cdot 92: 1$ to $1 \cdot 68: 1)$. The mean +2 SD in the symptomatic group was $1 \cdot 68: 1$ and was significantly raised $(P<0.01)$ over the $1 \cdot 40: 1$ of the controls. Of these 60 patients with low back pain 36 (group A) with a mean age of 41 (range 19-68) years had normal scintiscans, while 24 (group B) with a mean age of 34 (range 18-69) years had abnormal results. In the latter group 18 patients had bilateral scan abnormalities, while 6 had unilateral abnormalities. The unilaterally abnormal results were considered individually and were not averaged with the normal side.

In the physical examination of group A 3 patients $(8 \%)$ had a significant scoliosis and $1(3 \%)$ had a leg length discrepancy greater than $1 \mathrm{~cm}$. For group $B$ the respective numbers were 0 and 1 (4\%). In group A $14(39 \%)$ had an abnormal Schober test $(<4 \mathrm{~cm}$ expansion of a $10 \mathrm{~cm}$ segment of lumbar spine), while in group B this number was $9(38 \%)$. In group A 14 patients (42\%) had positive findings when the sacroiliac joints were stressed, while in group B 17 patients $(71 \%$ ) showed positive clinical findings. Although these data suggest an association between scintigraphic results and clinical features in group B, a further assessment of the group showed that in 12 patients there was concordance between clinical and scan findings (both bilateral and unilateral sacroiliac disease), that in 5 clinical and scan abnormalities were discordant for example, uniateral scan abnormality and contralateral clinical abnormality), and that in 7 there was no clinical evidence of sacroiliac disease.

All patients had radiographs. In group A 4 patients had evidence of degenerative disease of the hip (with good clinical correlation). 2 had degenerative changes of the lumbar spine, and 1 had metastatic lytic lesions. All radiographs were normal in group B with the exception of 1 patient who had grade III bilateral sacroiliitis.

An obstetrical review revealed a mean parity in group A of 1.83 births (both live and still births of more than 7 months gestation) and in group $A$ of 2.09 (group B does include 1 patient who is gravida 14). None of the patients had been pregnant in the 12 months preceding the initial scan. Persistent gastrointestinal symptoms were found in $10(28 \%)$ patients in group A and $9(38 \%)$ in group B, and these were primarily related to constipation. A history of recurrent urinary tract infections (fewer than 3 in the past 5 years) was found in $7(19 \%)$ patients in group A and $3(13 \%)$ in group B. Abnormal urine analyses paralleled these symptoms in the 2 groups (6 and 3 patients with pyuria respectively for groups A and B). No patient had a history of nephrolithiasis.

Laboratory abnormalities for the 2 groups are illustrated in Table 1. In addition the HLA B27 antigen was present in $5(14 \%)$ patients from groug $A$ and $3(13 \%)$ from group B. The frequency of this antigen in a series of normal patients from this region is $6 \%$.

Of the 60 patients in groups A and B 18 had repeat scintiscans performed within 6 months of the initial visit when a reasonable course of anti-inflammatory therapy had elapsed. All patients were treated with nonsalicylate, nonsteroidal anti-inflammatory agents, primarily with indomethacin. Four patients with initially normal SI:S ratios (mean 1.20:1) had repeat scans performed. These four (all of whom were still symptomatic) remained in the normal range at the time of the repeat scan. Seven patients who had mean values of $1 \cdot 48: 1$ prior to treatment remained symptomatic at the second assessment, and their mean ratios remained within the abnormal range at $1 \cdot 43: 1$. However, 7 patients who had symptomatic improvement showed mean ratios

Table 1 Laboratory values

\begin{tabular}{lll}
\hline & Group $A$ & Group B \\
\hline Hb $<12 \cdot 0 \mathrm{~g} / \mathrm{dl}$ & 0 & 1 \\
Leucocytes $<4 \times 109 / 1$ & 1 & 0 \\
ESR $>20 \mathrm{~mm} / \mathrm{h}$ & 9 & 7 \\
RF (+) & 0 & 1 \\
Raised alkaline & & 0 \\
$\quad$ phosphatase & 1 & 0 \\
Raised Ca & 0 & 0 \\
Raised PO & 0 & 3 \\
HLA B27 & 5 &
\end{tabular}

$\mathbf{R F}=$ rheumatoid factor. 
Table 2 Evolution of the scintiscan in 18 patients at follow-up

\begin{tabular}{lll}
\hline Patients & $\begin{array}{l}\text { Initial scan ratio } \\
(\text { mean } S I: S)\end{array}$ & $\begin{array}{l}\text { Follow-up scan ratio } \\
(\text { mean } S I: S)\end{array}$ \\
\hline 4 & $1 \cdot 20: 1$ & $1 \cdot 28: 1$ \\
& (symptomatic) & (symptomatic) \\
7 & $1 \cdot 48: 1$ & (symptomatic) \\
7 & (symptomatic) & $1 \cdot 28: 1$ \\
& $1 \cdot 51: 1$ & (asymptomatic) \\
\hline
\end{tabular}

SI :S = sacroiliac to sacral ratio.

of uptake falling from $1 \cdot 51: 1$ to $1 \cdot 28: 1$ over the follow-up period (Table 2).

\section{Discussion}

These data amplify previous results obtained using radionuclide bone scanning in the assessment of low back pain in women and lend support to an organic cause of pain which may not be evident on plain radiographs. ${ }^{4}$ In the symptomatic patients with abnormal scans the radiographs of the sacroiliac joints were normal in all but 1 , who had classical ankylosing spondylitis with the HLA B27 antigen.

Typically, the symptomatic patients and the controls were young women. The former group was, however, somewhat older, with a mean age of 38 compared with 31 for the controls. Within the symptomatic group those with scintiscan abnormalities tended to be younger (mean age 34) than those with normal scans (mean age 41). None of these differences was significant.

In our patient histories, clinical examinations, and laboratory studies we have attempted to uncover in those patients with QSS abnormalities any associated clinical and laboratory features or any other diseases known to be associated with sacroiliac disease. Viewed individually there were no specific historic, clinical, or laboratory results that distinguished group A from group B. Our data did, however, allow us to discount or exclude several disorders that have been associated with sacroiliac disease and with low back pain. With the exception of the 1 patient who had radiographic evidence of sacroiliitis (and was HLA B27 positive), 23 of the 24 patients in group $B$ had normal radiographs. Although it has been shown that scintiscan abnormalities may antedate radiographic changes in ankylosing spondylitis, ${ }^{2} 6$ only long-term follow-up of our patients would exclude this possibility. In addition only $13 \%$ of group B was HLA B27 positive, which is not significantly higher than in a local control population. The absence of a cor- relation between the B27 antigen and clinical and and scan results suggests that the sacroiliac disease seen in these women is not related to the same genetic factor as in ankylosing spondylitis. By history we were able to eliminate sacroiliac disease associated with Reiter's syndrome, ulcerative colitis, and Crohn's disease. The absence of cutaneous psoriasis and of peripheral joint and nail pathology makes the sacroiliitis of psoriatic arthropathy an unlikely cause.

While infectious and metabolic bone disorders may also produce sacroiliac disease, the clinical, radiographic, and laboratory results in our patients would make these unlikely aetiological factors. Only in group A did we find a patient with scintiscan evidence of back pain from metastatic sources. No patient in either group showed clinical evidence of nerve root compression, and radiographic evidence of degenerative disease of the lumbar spine was found only group A.

Our search included an inquiry into and examination of possible mechanical factors that could explain sacroiliac inflammation. Leg length discrepancies and the presence of scoliosis did not correlate with scintiscan abnormalities. None of our patients had a history of severe trauma resulting in a pelvic or lumbar spinal injury. It was also speculated that the ligamentous laxity that develops during recurrent pregnancies might contribute to sacroiliac instability and pain. The parity of both groups A and B was similar, however.

Previous studies have shown a correlation between scintiscan abnormalities and the radiographic changes of ankylosing spondylitis and of other disorders. ${ }^{7-9}$ Other studies have indicated the value of the scan in detecting sacroiliac disease where radiographs are normal. ${ }^{1-3}$ Caution in the interpretation of scintiscan data has been emphasised. ${ }^{10} 11$ We feel that those disorders, apart from sacroiliac disease, found by Ho et al. to yield a positive scintiscan result (namely, rheumatoid arthritis, degenerative disease of the lumbrosacral spine, and metabolic bone disease) have been excluded in our patients with positive QSS results. The progression of radiographs from normal to abnormal in patients whose scans were the only initial objective abnormality and the return to normal of scan activity following anti-inflammatory treatment in some patients suggests that abnormal scans may reflect sacroiliac inflammation. ${ }^{26}$

Our results indicate that sacroiliac disease is a frequent cause of organic low back pain in women. In searching for possible associations with abnormal scintigraphy we found that clinical evidence of a sacroiliac disorder did not necessarily parallel scan 
evidence of inflammation. In addition factors such as leg length discrepancy, associated radiological features, laboratory variables, and a significant obstetrical, gastrointestinal, or urinary tract history did not correlate with scintigraphic evidence of sacroiliac disease. Consequently the only clinical measure that we could use for follow-up was an assessment of the severity of symptoms. Symptomatic improvement in some of our patients which correlated with improvement in radionuclide scanning results would support the view that the scan is measuring intrinsic sacroiliac joint inflammation rather than a phenomenon secondary to mechanical causes. Although the aetiology of this inflammation is not clear, the disorder will respond to treatment with anti-inflammatory agents in some cases.

\section{References}

1 Lentle B C, Russell A S, Percy J S, Jackson F I. The scintigraphic investigation of sacroiliac disease. $J$ Nucl Med Allied Sci 1977: 18: 529-33.

2 Namey T C, McIntyre J, Buse M, LeRoy E C. Nucleographic studies of axial sponarthritides. Arthritis Rheum 1977; 20 : 1058-64.
3 Goldberg R P, Genant H K, Shimshak R, Shames D. Applications and limitations of quantitative sacroiliac joint scintigraphy. Radiology 1978; 128: 683-6.

4 Davis P, Lentle B C. Evidence for sacroiliac disease as a common cause of low backache in women. Lancet 1978; ii: 496-7.

5 Bennett R H J, Burch T A. New York symposium on population studies in the rheumatic diseases; new diagnostic criteria. Bull Rheum Dis 1967; 17: 453-8.

- Chalmers I M, Lentle B C, Percy J S. Russell A S. Sacroiliitis detected by bone scintiscanning: a clinical, radilogical, and scintigraphic follow-up study. Ann Rheum Dis 1979; 38: 112-7.

7 Barraclough D, Russell A S, Percy J S. Psoriatic spondylitis: a clinical, radiological, and scintiscan survey. $J$ Rheumatol 1977; 4: 282-7.

8 Russell A S, Davis P, Percy J S, Lentle B C. The sacroiliitis of acute Reiter's syndrome. $J$ Rheumatol 1977; 4: 293-6.

9 Davis P, Thomson A B R, Lentle B C. Quantitative sacroiliac scintigraphy in patients with Crohn's disease. Arthritis Rheum 1978; 21 : 234-7.

10 Lugon M, Torode A S, Travers R L, Amaral H, Lavender J P, Hughes G R V. Sacro-iliac joint scanning with technetium-99 diphosphonate. Rheumatol Rehabil 1979; 18: 131-6.

11 Ho G, Sadovnikoff N, Malhotra C M, Claunch B C Quantitative sacroiliac joint scintigraphy: a clinical assessment. Arthritis Rheum 1979; 22: 837-44. 